\title{
A Survey on the Methods of Super-Resolution Image Reconstruction
}

\author{
R. Sudheer Babu \\ Assistant Professor, \\ ECE Department, \\ G.Pullareddy Engineering College, \\ Kurnool-518007, A.P. INDIA
}

\author{
Dr.K.E.Sreenivasa Murthy \\ Principal, \\ Sri Venkateswara Institute of Technology, \\ Rapthadu, Anantapur, INDIA
}

\begin{abstract}
Super-resolution (SR) image reconstruction is the process of combining several low resolution images into a single higher resolution image. There is a driving need for digital images of higher resolutions and quality. However, there is a limit to the spatial resolution that can be recorded by any digital device. Growing interest in super-resolution (SR) restoration of video sequences and the closely related problem of construction of SR still images from image sequences has led to the emergence of several competing SR reconstruction methodologies. In this paper, the principle of super-resolution image reconstruction and several state-of-the-art SR reconstruction methods were introduced. We critique these methods and at last, several aspects of super-resolution image reconstruction that should be studied further more were put forward
\end{abstract}

Key Words: Image Reconstruction, Super Resolution, Finite Support, Deconvolution, Denoise.

\section{INTRODUCTION}

Super-resolution, loosely speaking, is the process of recovering a high-resolution image from a set of lowresolution input images. Any given set of source low resolution (LR) images only captures a finite amount of information from a scene; the goal of SR is to extract the independent information from each image in that set and combine the information into a single high resolution (HR) image. The only requirement is that each LR image must contain some information that is unique to that image. This means that when these LR images are mapped onto a common reference plane their samples must be subpixel shifted from samples of other images - otherwise the images would contain only redundant information and SR reconstruction would not be possible. Most methods in SR are strictly reconstruction based; that is, they are based primarily on uniform and nonuniform sampling theorems and do not attempt to create any information not found in the LR images. There are also learning SR methods that create new information based on generative models.

SR techniques can prove useful in many different applications, and these applications can have different requirements in terms of both quality and computational complexity. The quality may also vary for different methods based on characteristics of the input image. The implementation complexity may be affected by implementation specifics, such as the availability of specific optimized libraries. Finally the artifacts caused by poor SR performance can be more visually distracting than blurring from interpolation. For these and other reasons choosing between SR methods is a complex task.
A variety of approaches for solving the super-resolution problem have been proposed. Initial attempts worked in the frequency domain, typically recovering higher frequency components by taking advantage of the shifting and aliasing properties of the Fourier transform. Deterministic regularization approaches, which work in the spatial domain, enable easier inclusion of a priori constraints on the solution space (typically with smoothness prior). Stochastic methods have received the most attention lately as they generalize the deterministic regularization approaches and enable more natural inclusion of prior knowledge. Other approaches include non-uniform interpolation, projection onto convex sets, iterative back projection, and adaptive filtering. With the increased emphasis on stochastic techniques has also come increased emphasis on learning priors from from example data rather than relying on more heuristically derived information.

The following sections will hopefully serve to elucidate the super- resolution problem. We start by introducing basic principle of super resolution image reconstruction , then cover various approaches to the super-resolution problem frequency domain techniques, spatial domain techniques which covers, non-uniform interpolation, deterministic regularization, stochastic methods, projection onto con- vex sets, and iterative back projection. Next we cover recent work attempting to determine limits to super-resolution techniques. Finally, we conclude with a section on potential future research directions.

\section{PRINCIPLE OF SUPER-RESOLUTION IMAGE RECONSTRUCTION}

\subsection{Definition of Super Resolution}

Non-coherent transfer function of an optical system is the autocorrelation of its pupil function, which means that the transfer function is necessarily band-limited. In another way, the value of transfer function should be zero when frequency determined by diffraction limit is above certain value. Apparently, de-convolution can only restore the spectrum of object to diffraction limit and cannot surpass it. However, by using Fourier transformation, we can get resolution above diffraction limit in theory. The restoration technology which is trying to restore the information above diffraction limit is called Super-resolution techniques. And the methods used in these techniques can be called Extrapolation of Bandlimited. Diffraction limiting Images of space objects can be obtain through high-resolution restoration of speckle images of these objects. But, with super-resolution information, resolution can be improved further by restoration and reconstruction of near- diffraction-limit images. 


\subsection{Principle}

Super-resolution image reconstruction is based on the theory of Analytic Continuation, which means reconstruction of the whole analytic function according to its values in certain area. Because of diffraction of lights, spectrum distribution of certain image is infinite in space and optical system truncates its frequency to obtain frequency-truncated image that is finite in space. Generally, truncation function cannot be bandlimited, but a diffraction limited optical system's truncation is band- limited, therefore, the reconstruction of whole spectrum function or just spectrum function above certain frequency is possible.

Assume the imaging model:

$$
g(x, y)=h(x, y) * f(x, y)+n(x, y)
$$

Where, $h(x, y)$ is the point spread function (PSF), ideal image, $\mathrm{g}(\mathrm{x}, \mathrm{y})$ is the original image and $\mathrm{n}(\mathrm{x}, \mathrm{y})$ is the noise.

Its Fourier transformation is:

$$
G(u, v)=H(u, v) \cdot F(u, v)+N(u, v)
$$

Super-resolution reconstruction is to perform analytic continuation to $F(u, v)$ to extend its support domain by using prior information of objects and posterior processing technologies, and then get a new PSF $H^{\prime}(u, v) . H(u, v)$ also has the extended support domain, thus the resolution of image is improved.

\section{FREQUENCY DOMAIN METHODS}

A major class of SR methods utilize a frequency domain formulation of the SR problem. Frequency domain methods are based on three fundamental principles: i) the shifting property of the Fourier transform (FT), ii) the aliasing relationship between the continuous Fourier transform (CFT) and the discrete Fourier transform (DFT), iii) the original scene is band-limited. These properties allow the formulation of a system of equations relating the aliased DFT coefficients of the observed images to samples of the CFT of the unknown scene. These equations are solved yielding the frequency domain coefficients of the original scene, which may then be recovered by inverse DFT. Formulation of the system of equations requires knowledge of the translational motion between frames to sub-pixel accuracy. Each observation image must contribute independent equations, which places restrictions on the inter-frame motion that contributes useful data.

Denote the continuous scene by $f(x, y)$. Global translations yield $\mathrm{R}$ shifted images, $f_{r}(x, y)=f\left(x+\Delta x_{r}, y+\Delta y_{r}\right), r=1,2, \ldots R . \quad$ The CFT of the scene is given by $F(u, v)$ and that of the translations by $\operatorname{Fr}(u, v)$. The shifted images are impulse sampled to yield observed images $y_{r}[m, n]=f\left(m T_{x}+\Delta x_{r}, n T_{y}+\Delta y_{r}\right)$ with $m=0,1,2 \ldots . M-1$ and $n=0,1,2 \ldots . . N-1$. The $\mathrm{R}$ corresponding 2D DFT's are denoted by $y_{r}[k, l]$. The CFT of the scene and the DFT's of the shifted and sampled images are related via aliasing,

$$
y_{r}[k, l]=\alpha \sum_{p=-\infty}^{\infty} \sum_{q=-\infty}^{\infty} F_{r}\left(\frac{k}{M T_{x}}+p f_{s_{x}}, \frac{l}{N T_{y}}+q f_{s_{y}}\right)
$$

where $f_{s_{x}}=1 / T_{x}$ and $f_{s_{y}}=1 / T_{y}$ are the sampling rates in the $x$ and $y$ dimensions respectively and $\alpha=1 /\left(T_{x} T_{y}\right)$. The shifting property of the CFT relates spatial domain translation to the frequency domain as phase shifting as,

$$
F_{r}(u, v)=e^{j 2 \pi\left(\Delta x_{r} u+\Delta y_{r} v\right)} F(u, v)
$$

If $f(x, y)$ is band-limited, $\exists L_{u}, L_{v}$ s.t. $F(u, v) \rightarrow 0$ for $|u| \geq L_{u} f_{s_{x}}$ and $|v| \geq L_{u} f_{s_{y}}$. Assuming $f(x ; y)$ is band limited, we may use (4) to rewrite the alias relationship (3) in matrix form as,

$$
Y=\phi F
$$

$\mathrm{Y}$ is a $R \times 1$ column vector with the $\mathrm{rth}$ element being the DFT coefficients $\operatorname{Yr}[k, l]$ of the observed image $Y_{r}[m, n] . \phi$ is a matrix which relates the DFT of the observation data to samples of the unknown CFT of $f(x, y)$ contained in the $4 L_{u} L_{v} \times 1$ vector $\mathrm{F} . \mathrm{SR}$ reconstruction thus requires finding the DFT's of the R observed images, determining $\phi$ (motion estimation), solving the system of equations (5) for $\mathrm{F}$ and applying the inverse DFT to obtain the reconstructed image. Several extensions to the basic Tsai-Huang method have been proposed. A LSI blur PSF is included in [17] and the equivalent of (5) is solved using a least squares approach to mitigate the effects of observation noise and insufficient observation data. A computationally efficient recursive least squares (RLS) solution for (5) is proposed in [10] and extended with a Tikhonov regularization solution method in [11] in an attempt to address the ill-posedness of SR inverse problem. Robustness to errors in observations as well as motivated the use of a total least squares (TLS) approach in [1] which is implemented using a recursive algorithm for computational efficiency. Techniques based on the multichannel sampling theorem [2] have also been considered [21].

Though implemented in the spatial domain, the technique is fundamentally a frequency domain method relying on the shift property of the Fourier transform to model the translation of the source imagery. Frequency domain SR methods provide the advantages of theoretical simplicity, low computational complexity, are highly amenable to parallel implementation due to decoupling of the frequency domain equations (5) and exhibit an intuitive de-aliasing SR mechanism. Disadvantages include the limitation to global translational motion and space invariant degradation models (necessitated by the requirement for a Fourier domain analog of the spatial domain motion and degradation model) and limited ability for inclusion of spatial domain a-priori knowledge for regularization. 


\section{SPATIAL DOMAIN METHODS}

Approaching the super-resolution problem in the frequency domain makes a lot of sense because it is relatively simple and computationally efficient. However, there are some problems with a frequency domain formulation. For one, it restricts the inter-frame motion to be translational because the DFT assumes uniformly spaced samples. Another disadvantage is that prior knowledge that might be used to constrain or regularize the super-resolution problem is often difficult to express in the frequency domain. Since the super-resolution problem is fundamentally ill-posed4, incorporation of prior knowledge is essential to achieve good results. A variety of techniques exist for the super-resolution problem in the spatial domain. These solutions include interpolation, deterministic regularized techniques, stochastic methods, iterative back projection, and projection onto convex sets among others. The primary advantages to working in the spatial domain are support for unconstrained motion between frames and ease of incorporating prior knowledge into the solution.

\subsection{Image Interpolation Algorithms 4.1.1 Bicubic Interpolation}

Given a sampled signal, its continuous counterpart can be approximated using some suitable interpolation kernel. 2D interpolation is usually accomplished by applying successively 1D kernel interpolation on horizontal and vertical directions. For uniformly spaced data, the continuous-domain signal $Y(u, v)$ can be written as,

$$
Y(u, v)=\sum_{k} \sum_{l} y(k, l) h\left(\frac{u-u_{k}}{\Delta u}\right) h\left(\frac{v-v_{k}}{\Delta v}\right)
$$

In this expression, $(\Delta u, \Delta v)$ are sampling intervals, $h()$ is the interpolation kernel and $\{\mathrm{y}(\mathrm{k}, \mathrm{l})\}$ represent the pixel array in the Low rsolution(LR) grid. The SR signal is obtained by resampling (6) on a finer grid. In [5], the cubic convolution kernel is given as,

$h(s)=\left\{\begin{array}{lc}1.5|\mathrm{~s}|^{3}-2.5|\mathrm{~s}|^{2}+1 & 0 \leq|\mathrm{s}|<1 \\ -0.5|\mathrm{~s}|^{3}+2.5|\mathrm{~s}|^{2}-4|\mathrm{~s}|+2 & 1 \leq|\mathrm{s}|<2 \\ 0 & 2 \leq|\mathrm{s}|\end{array}\right.$

\subsubsection{Interpolation of Non-Uniformly Spaced Samples}

Registering a set of LR images using motion compensation results in a single, dense composite image of non-uniformly spaced samples. A SR image may be reconstructed from this composite using techniques for reconstruction from nonuniformly spaced samples. Restoration techniques are sometimes applied to compensate for degradations [17]. Iterative reconstruction techniques, based on the Landweber iteration, have also been applied [12]. Such interpolation methods are unfortunately overly simplistic. Since the observed data result from severely under sampled, spatially averaged areas, the reconstruction step (which typically assumes impulse sampling) is incapable of reconstructing significantly more frequency content than is present in a single LR frame. Degradation models are limited, and no a- priori constraints are used. There is also question as to the optimality of separate merging and restoration steps.

\subsubsection{Wavelet-Based Image Interpolation}

Wavelet-based image interpolation methods assume that the available image is the coarse approximation $\left(L L_{0}\right)$, that is, low-pass filtered subband of an HR image. The interpolation methods then first try to recover the missing horizontal $\left(\mathrm{LH}_{0}\right)$, vertical $\left(H L_{0}\right)$ and diagonal $\left(H H_{0}\right)$ detail subbands, and then obtain the HR image by taking the inverse Discrete Wavelet Transform (DWT) of the expanded image. An important property of DWT is the persistence property. In fact several wavelet-based compression schemes, such as embedded zero tree wavelets, employ this property. Temizel and Vlachos [6] proposed a wavelet based image interpolation method. They used the idea of "persistence", which implies that the magnitudes of wavelet coefficients corresponding to the same spatial location tend to propagate from lower scales to higher resolution scales. They extended the "persistence" idea to correlation coefficients. First, one goes one scale down, and estimates $\left(H L_{l}\right)$ by high pass filtering $\left(L L_{l}\right)$ horizontally. Then, correlation coefficients between $\left(H L_{l}\right)$ and its estimate are computed. Using these correlation coefficients and estimate of $\left(H L_{0}\right)$, exact value of $\left(H L_{0}\right)$ is computed. All these horizontal and vertical filtering operations are, however, implemented without decimation, in other words one stays at the resolution level of $\left(L L_{0}\right)$. The $\left(H H_{0}\right)$ is not predicted since it is judged visually less informative, so that the corresponding coefficients in the inverse DWT are filled with zeros.

\subsubsection{Edge Adaptive Image Interpolation}

The imaging process and the concomitant loss of resolution are modeled as low-pass filtering and decimation stages in [7]. The low-pass filtering operation modifies the values of the pixels near the edges proportionally to the distance between pixels and the edge. Therefore the analysis of the low resolution pixels should give an idea about the position of the edge at sub-pixel level. The one-dimensional case is illustrated in Fig.1

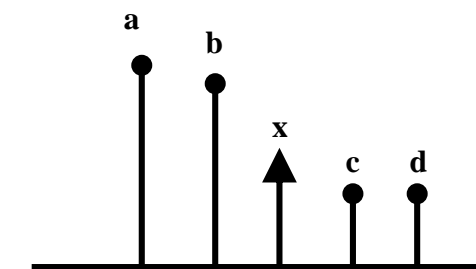

Fig - 1 : The $x$ pixel, astride an edge, is to be interpolated.

The interpolated value $\mathrm{x}$ between the given $\mathrm{a}, \mathrm{b}, \mathrm{c}, \mathrm{d}$ neighbors become [9]:

$$
\begin{aligned}
& x=\mu b+(1-\mu) c \\
& \mu=\frac{k(c-d)^{2}+1}{k\left((a-b)^{2}+(c-d)^{2}\right)+2}
\end{aligned}
$$

where $k$ is an input parameter and affects the edge sensitivity. When edge is in midway between $b$ and $c, a b=c-d$ and $x=(b+c) / 2$. When edge is closer to $c$, then $a b<c$ - $d$ and $x$ takes a value closer to $b$. In $2 \mathrm{D}$, first the missing pixels along the rows and the missing pixels along the columns are estimated, separately. Then the diagonal pixels are estimated using the results of the previous steps and the mean value of the two results is taken. 


\subsection{Iterated Backprojection}

Given a SR estimate $\hat{z}$ and the imaging model $\mathrm{H}$, it is possible to simulate the LR images $Y$ as $Y=H \hat{z}$. Iterated backprojection (IBP) procedures update the estimate of the SR reconstruction by backprojecting the error between the $j^{\text {th }}$ simulated LR images $Y j$ and the observed LR images $Y$ via the backprojection operator $\mathrm{H}^{\mathrm{BP}}$ which apportions "blame" to pixels in the $\mathrm{SR}$ estimate $\hat{z} j$. Typically $\mathrm{H}^{\mathrm{BP}}$ approximates $H^{-1}$ algebraically,

$$
\begin{array}{r}
\hat{z}(j+1)=\hat{z}(j)+H^{B P}(Y-Y(j)) \\
=\square^{\prime}(j)+H^{B P}(Y-H \hat{z}(j))
\end{array}
$$

Equation (9) is iterated until some error criterion dependent on $Y, Y(j)$ is minimized. Application of the IBP method may be found in [9]. IBP enforces that the SR reconstruction match (via the observation equation) the observed data. Unfortunately the SR reconstruction is not unique since SR is an ill-posed inverse problem. Inclusion of a-priori constraints is not easily achieved in the IBP method.

\subsection{Stochastic SR Reconstruction Methods}

Stochastic methods (Bayesian in particular) which treat SR reconstruction as a statistical estimation problem have rapidly gained prominence since they provide a powerful theoretical framework for the inclusion of a-priori constraints necessary for satisfactory solution of the ill-posed SR inverse problem. The observed data $Y$, noise $N$ and SR image $\mathrm{z}$ are assumed stochastic. Consider now the stochastic observation equation $Y=H z+N$. The Maximum

A-Posteriori (MAP) approach to estimating $z$ seeks the estimate $\hat{z}_{M A P}$ for which the a-posteriori probability, $\operatorname{Pr} z \mid Y$ is a maximum.

Formally, we seek $\hat{z}$ MAP as,

$$
\begin{aligned}
\hat{z}_{M A P} & =\arg \max _{z}[\operatorname{Pr}\{z \mid Y\}] \\
& =\arg \max _{z}[\log \operatorname{Pr}\{Y \mid z\}+\log \operatorname{Pr}\{z\}]
\end{aligned}
$$

The second line is found by applying Bayes' rule, recognizing that $\hat{z}_{M A P}$ is independent of $\operatorname{Pr}\{Y\}$ and taking logarithms. The term $\log \operatorname{Pr}\{\mathrm{Y} \mid \mathrm{z}\}$ is the log-likelihood function and $\operatorname{Pr}\{z\}$ is the a-priori density of $z$. Since $Y=H z+N$, the likelihood function is determined by the PDF of the noise as $\operatorname{Pr}\{\mathrm{Y} \mid \mathrm{z}\}=\mathrm{f}_{N}(Y-H z)$. It is common to utilize

Markov random field (MRF) image models as the prior term Pr fzg. Under typical assumptions of Gaussian noise the prior may be chosen to ensure a convex optimization in (5) enabling the use of descent optimization procedures. Examples of the application of Bayesian methods to SR reconstruction may be found in [15] using a Huber MRF and [3,7] with a Gaussian MRF. Maximum likelihood (ML) estimation has also been applied to SR reconstruction [18].
ML estimation is a special case of MAP estimation (no prior term). Since the inclusion of a-priori information is essential for the solution of ill-posed inverse problems, MAP estimation should be used in preference to ML. A major advantage of the Bayesian framework is the direct inclusion of a-priori constraints on the solution, often as MRF priors which provide a powerful method for image modeling using (possibly non-linear) local neighbor interaction. MAP estimation with convex priors implies a globally convex optimization, ensuring solution existence and uniqueness allowing the application of efficient descent optimization methods. Simultaneous motion estimation and restoration is also possible [7]. The rich area of statistical estimation theory is directly applicable to stochastic SR reconstruction methods.

\subsection{Set Theoretic Reconstruction Methods}

Set theoretic methods, especially the method of projection onto convex sets (POCS), are popular as they are simple, utilize the powerful spatial domain observation model, and allow convenient inclusion of a priori information. In set theoretic methods, the space of SR solution images is intersected with a set of (typically convex) constraint sets representing desirable SR image characteristics such as positivity, bounded energy, fidelity to data, smoothness etc., to yield a reduced solution space. POCS refers to an iterative procedure which, given any point in the space SR images, locates a point which satisfies all the convex constraint sets. Convex sets which represent constraints on the solution space of $\mathrm{z}$ are defined. Data consistency is typically represented by a set $\left\{z:|Y-H z|<\delta_{0}\right\}$, positivity by $\left\{z: z_{i}>0 \forall i\right\}$, bounded energy by $\{z:\|z\| \leq E\}$, compact support $\left\{z: z_{i}=0, i \in A\right\}$ and so on. For each convex constraint set so defined, a projection operator is determined.

The projection operator $P_{\alpha}$ associated with the constraint set $C_{\alpha}$ projects a point in the space of $z$ onto the closest point on the surface of $C_{\alpha}$. It can be shown that repeated application of the iteration $z^{(n+1)}=P_{1} P_{2} P_{3} \ldots \ldots P_{K} z^{(n)}$ will result in convergence to a solution on the surface of the intersection of the $\mathrm{K}$ convex constraints sets. Note that this point is in general non-unique and is dependent on the initial guess. POCS reconstruction methods have been successfully applied to sophisticated observation and degradation models $[13,6]$. An alternate set theoretic SR reconstruction method [19] uses an ellipsoid to bound the constraint sets. The centroid of this ellipsoid is taken as the SR estimate. Since direct computation of this point is infeasible, an iterative solution method is used. The advantages of set theoretic SR reconstruction techniques were discussed at the beginning of this section. These methods have the disadvantages of non-uniqueness of solution, dependence of the solution on the initial guess, slow convergence and high computational cost. Though the bounding ellipsoid method ensures a unique solution, this solution is has no claim to optimality.

\subsection{Hybrid ML/MAP/POCS Methods}

Work has been undertaken on combined ML/MAP/POCS based approaches to $\mathrm{SR}$ reconstruction $[15,5]$. The desirable characteristics of stochastic estimation and POCS are combined in a hybrid optimization method. The a-posteriori 
density or likelihood function is maximized subject to containment of the solution in the intersection of the convex constraint sets.

\subsection{Optimal and Adaptive Filtering}

Inverse filtering approaches to SR reconstruction have been proposed, however these techniques are limited in terms of inclusion of a-priori constraints as compared with POCS or Bayesian methods and are mentioned only for completeness. Techniques based on adaptive filtering, have also seen application in SR reconstruction [14, 4]. These methods are in effect LMMSE estimators and do not include non-linear apriori constraints.

\subsection{TikhonovArsenin Regularization}

Due the illposedness of SR reconstruction, Tikhonov-Arsenin regularized SR reconstruction methods have been examined [8]. The regularizing functional characteristic of this approach are typically special cases of MRF priors in the Bayesian framework.

\section{SUMMARY AND COMPARISON}

A general comparison of frequency and spatial domain SR reconstructions methods is presented in Table 1.

\begin{tabular}{|l|l|l|}
\hline & \multicolumn{1}{|c|}{ Freq. Domain } & \multicolumn{1}{c|}{ Spat. Domain } \\
\hline Observation model & Frequency domain & Spatial domain \\
\hline Motion models & Global translation & Almost unlimited \\
\hline SR Mechanism & Limited, LSI & LSI or LSV \\
\hline Noise model & Limited, SI & Very Flexible \\
\hline Degradation model & De-aliasing & De-aliasing A-priori info \\
\hline SR Mechanism & Low & High \\
\hline Computation req. & Limited & Good \\
\hline A-priori info & Limited & Excellent \\
\hline Regularization & Poor & Excellent \\
\hline App. performance & Limited & Wide \\
\hline Applicability & Good & Almost unlimited \\
\hline
\end{tabular}

Table 1. Frequency vs. spatial domain SR

Spatial domain SR reconstruction methods, though computationally more expensive, and more complex than their frequency domain counterparts, offer important advantages in terms of flexibility. Two powerful classes of spatial domain methods; the Bayesian (MAP) approach and the set theoretic POCS methods are compared in Table 2.

\begin{tabular}{|l|l|l|}
\hline & \multicolumn{1}{|c|}{ Bayesian (MAP) } & \multicolumn{1}{c|}{ POCS } \\
\hline Applicable theory & Vast & Limited \\
\hline A-priori info & $\begin{array}{l}\text { Prior PDF } \\
\text { Easy to incorporate, No hard } \\
\text { constraints }\end{array}$ & $\begin{array}{l}\text { Convex sets } \\
\text { Easy to incorporate } \\
\text { Powerful yet simple }\end{array}$ \\
\hline SR solution & $\begin{array}{l}\text { MAP estimate } \\
\text { Unique }\end{array}$ & $\begin{array}{l}\text { Non-uniqueness } \\
\text { of constraint sets }\end{array}$ \\
\hline Computation req. & Iterative & High \\
\hline Convergence & Good & Possibly slow \\
\hline Optimization & High & Iterative \\
\hline Complications & $\begin{array}{l}\text { Non-convex priors Optimization } \\
\text { under }\end{array}$ & $\begin{array}{l}\text { Operators } \\
\text { Defn. of projection }\end{array}$ \\
\hline
\end{tabular}

Table 2. MAP vs. POCS SR

\section{FUTURE RESEARCH DIRECTIONS}

Three research areas promise improved SR methods:

Motion Estimation: SR enhancement of arbitrary scenes containing global, multiple independent motion, occlusions, transparency etc. is a focus of SR research. Achieving this is critically dependent on robust, model based, sub-pixel accuracy motion estimation and segmentation techniques presently an open research problem. Motion is typically estimated from the observed undersampled data the reliability of these estimates should be investigated.

Simultaneous multi-frame motion estimation should provide performance and reliability improvements over common two frame techniques. For non-parametric motion models, constrained motion estimation methods which ensure consistency in motion maps should be used. Regularized motion estimation methods should be utilized to resolve the ill-posedness of the motion estimation problem. Sparse motion maps should be considered. Sparse maps typically provide accurate motion estimates in areas of high spatial variance exactly where SR techniques deliver greatest enhancement.

Reliability measures associated with motion estimates should enable weighted reconstruction. Global and local motion models, combined with iterative motion estimation, identification and segmentation provide a framework for general scene SR enhancement. Independent model based motion predictors and estimators should be utilized for independently moving objects. Simultaneous motion estimation and SR reconstruction approaches should yield improvements in both motion estimates and SR reconstruction. 
Degradation Models: Accurate degradation (observation) models promise improved SR reconstructions. Several SR application areas may benefit from improved degradation modeling. Only recently has color SR reconstruction been addressed [16]. Improved motion estimates and reconstructions are possible by utilizing correlated information in color bands. Degradation models for lossy compression schemes (color subsampling and quantization effects) promise improved reconstruction of compressed video. Similarly, considering degradations inherent in magnetic media recording and playback are expected to improve SR reconstructions from low cost camcorder data. The response of typical commercial CCD arrays departs considerably from the simple integrate and sample model prevalent in much of the literature. Modeling of sensor geometry, spatio-temporal integration characteristics, noise and readout effects promise more realistic observation models which are expected to result in SR reconstruction performance improvements.

Restoration Algorithms: MAP and POCS based algorithms are very successful and to a degree, complimentary. Hybrid MAP/POCS restoration techniques will combine the mathematical rigor and uniqueness of solution of MAP estimation with the convenient a-priori constraints of POCS. The hybrid method is MAP based but with constraint projections inserted into the iterative maximization of the a-posteriori density in a generalization of the constrained MAP optimization of [15]. Simultaneous motion estimation and restoration yields improved reconstructions since motion estimation and reconstruction are interrelated. Separate motion estimation and restoration, as is commonly done, is sub-optimal as a result of this interdependence. Simultaneous multi-frame SR restoration is expected to achieve higher performance since additional spatio-temporal constraints on the SR image ensemble may be included. This technique has seen limited application in SR reconstruction.

\section{REFERENCES}

[1] N. K. Bose, H. C. Kim, and H. M. Valenzuela. Recursive Total Least Squares Algorithm for Image Reconstruction from Noisy, Undersampled Multiframes. Multidimensional Systems and Signal Processing, 4(3):253-268, July 1993.

[2] J. L. Brown. Multichannel sampling of low-pass signals. IEEE Trans. CAS, 28(2):101- 106, 1981.

[3] P. Cheeseman, B. Kanefsky, R. Kraft, J. Stutz, and R. Han-son. Super-resolved surface reconstruction from multiple images. In Maximum Entropy and Bayesian Methods, pages 293-308. Kluwer, Santa Barbara, CA, 1996.

[4] M. Elad and A. Feuer. Super-Resolution Restoration of Continuous Image Sequence - Adaptive Filtering Approach. Submitted to IEEE Trans. IP.

[5] R. Keys, "Cubic convolution interpolation for digital image processing," IEEE Transactions on Acoustics, Speech, and Signal Processing, vol. 29, pp. 1153 - 1160, Dec 1981.

[6] P. E. Eren, M. I. Sezan, and A. Tekalp. Robust, ObjectBased High-Resolution Image Reconstruction from LowResolution Video. IEEE Trans. IP, 6(10):1446-1451, 1997.
[7] S. Carrato, G. Ramponi, S. Marsi, "A simple edge sensitive image interpolation filter," Image Processing, IEEE Proceedings, International Conference, vol. 3 pp.711 - 714, 16-19 Sept. 1996.

[8] M.C. Hong, M. G. Kang, and A. K. Katsaggelos. A regularized multichannel restoration approach for globally optimal high resolution video sequence. In SPIE VCIP, volume 3024, pages 1306-1316, San Jose, Feb. 1997.

[9] R. C. Hardie, K. J. Barnard, and E. E. Armstrong. Joint MAP Registration and High Resolution Image Estimation Using a Sequence of Undersampled Images IEEE Trans IP, 6(12):1621-1633, Dec. 1997.

[10] S. P. Kim, N. K. Bose, and H. M. Valenzuela. Recursive reconstruction of high resolution image from noisy under- sampled multiframes. IEEE Trans. ASSP, 38(6):1013-1027,1990.

[11] S. P. Kim and W.-Y. Su. Recursive high-resolution recon- struction of blurred multiframe images. IEEE Trans. IP, 2:534-539, Oct. 1993.

[12] T. Komatsu, T.Igarashi, K. Aizawa, and T. Saito. Very high resolution imaging scheme with multiple different aperture cameras. Signal Processing Image Communication, 5: 511-526, Dec. 1993.

[13] A. J. Patti, M. I. Sezan, and A. M. Tekalp. Superresolution Video Reconstruction with Arbitrary Sampling Lattices and Nonzero Aperture Time. IEEE Trans. IP, 6(8):1064-1076, Aug. 1997.

[14] A. J. Patti, A. M. Tekalp, and M. I. Sezan. A New Motion Compensated Reduced Order Model Kalman Filter for Space-Varying Restoration of Progressive and Interlaced Video. IEEE Trans. IP, 7(4):543-554, Apr.1998.

[15] R. R. Schultz and R. L. Stevenson. Extraction of highresolution frames from video sequences. IEEE Trans. IP, 5(6):996-1011, June 1996.

[16] N. R. Shah and A. Zakhor. Multiframe spatial resolution enhancement of color video. In ICIP, volume I, pages 985-988, Lausanne, Switzerland, Sept. 1996.

[17] A. M. Tekalp, M. K. Ozkan, and M. I. Sezan. Highresolution image reconstruction from lower-resolution image sequences and space-varying image restoration. In ICASSP, volume III, pages 169-172, San Francisco, 1992.

[18] B. C. Tom and A. K. Katsaggelos. Reconstruction of a high resolution image from multiple degraded misregistered low resolution images. In SPIE VCIP, volume 2308, pages 971-981, Chicago, Sept. 1994.

[19] B. C. Tom and A. K. Katsaggelos. An Iterative Algorithm for Improving the Resolution of Video Sequences. In SPIE VCIP, volume 2727, pages 1430 1438, Orlando, Mar. 1996.

[20] R. Y. Tsai and T. S. Huang. Multiframe image restoration and registration. In R. Y. Tsai and T. S Huang, editors, Advances in Computer Vision and Image Processing, volume 1, pages 317-339. JAI Press Inc., 1984.

[21] H. Ur and D. Gross. Improved resolution from subpixel shifted pictures. CVGIP: Graphical models and Image Processing, 54:181-186, Mar. 1992. 